\title{
Efecto del tratamiento con atorvastatina sobre la incidencia de eventos cardiovasculares y de diabetes
}

\author{
Effect of treatment with atorvastatin on the incidence of cardiovascular events and diabetes
}

Waters D y col. J. Am. Coll. Cardiol. 2013; 61:148-52.

\section{Objetivo}

Examinar la incidencia* de diabetes y de eventos cardiovasculares (ECV) asociada al uso de atorvastatina en prevención secundaria

\section{Diseño y población}

Análisis secundario* de los datos de los pacientes sin diagnóstico de diabetes al comienzo de dos ensayos clínicos aleatorizados, con una media de seguimiento de 4,85 años:

1) TNT ( $\mathrm{n}=7595): 10 \mathrm{mg} / \mathrm{día}$ vs. $80 \mathrm{mg} /$ día de atorvastatina en individuos de 35 a 75 años con enfermedad coronaria estable;

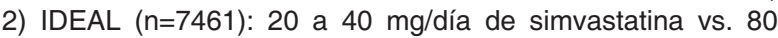
$\mathrm{mg} /$ día de atorvastatina en menores de 80 años con antecedentes de infarto agudo de miocardio (IAM).
Evaluación de los factores de riesgo y medición de resultados principales

Fueron clasificados como "bajo riesgo" quienes tenían menos de dos de los siguientes factores de riesgo y como "alto riesgo", quienes tenían dos o más: 1) glucemia mayor a $100 \mathrm{mg} / \mathrm{dL}$, 2) índice de masa corporal mayor a $30 \mathrm{~kg} / \mathrm{m} 2$, 3) trigliceridemia mayor a $150 \mathrm{mg} / \mathrm{dL}$, 4) diagnóstico de hipertensión arterial. Fueron identificados como "nuevos casos de diabetes" quienes desarrollaron durante el seguimiento dos glucemias en ayunas superiores a $126 \mathrm{mg} / \mathrm{dL}$, un registro $36 \mathrm{mg} / \mathrm{dL}$ por encima de su basal, y todo paciente en quien fuera reportado su desarrollo como efecto adverso. Los ECV incluidos en el análisis fueron: muerte coronaria, IAM, accidente cerebro-vascular y paro cardíaco resucitado.

\section{Resultados principales}

Se describen en la tabla 1.

Tabla 1. Incidencia de diabetes y de eventos cardiovasculares según el riesgo basal de diabetes y el tratamiento con estatinas asignado.

\begin{tabular}{|c|c|c|c|c|}
\hline \multirow[b]{2}{*}{ Resultado evaluado } & \multirow{2}{*}{$\begin{array}{l}\text { Riesgo basal } \\
\text { de desarrollo } \\
\text { de diabetes }\end{array}$} & \multicolumn{2}{|c|}{$\begin{array}{c}\text { Eventos/total (\%) } \\
\text { de acuerdo al tratamiento asignado (mg/dia) }\end{array}$} & \multirow[b]{2}{*}{$\begin{array}{l}\text { Razón de riesgo o HR } \\
\text { (IC95\%) }\end{array}$} \\
\hline & & Atorvastatina $\mathbf{8 0}$ & $\begin{array}{c}\text { Atorvastatina } 10 \\
\text { Sinvastatina } 20 \text { o } 40\end{array}$ & \\
\hline \multirow{3}{*}{ Nuevos casos de diabetes } & Bajo & $142 / 4407(3,2 \%)$ & $148 / 4418(3,4 \%)$ & $0,97(0,77$ a 1,22$)$ \\
\hline & Alto & $448 / 3128(14,3 \%)$ & $368 / 3103(11,9 \%)$ & $1,24(1,08$ a 1,42$)$ \\
\hline & Global & $590 / 7535(7,8 \%)$ & $516 / 7521(6,9 \%)$ & $1,16(1,03$ a 1,30$)$ \\
\hline \multirow{3}{*}{ Eventos cardio-vasculares } & Bajo & $375 / 4407(8,5 \%)$ & $433 / 4418(9,8 \%)$ & $0,87(0,76$ a 0,99$)$ \\
\hline & Alto & $315 / 3128(10,1 \%)$ & $373 / 3103(12,0 \%)$ & $0,82(0,71$ a 0,96$)$ \\
\hline & Global & $690 / 7535(9,2 \%)$ & $806 / 7521(10,7 \%)$ & $0,85(0,77$ a 0,94$)$ \\
\hline
\end{tabular}

\section{Conclusiones}

Sin olvidar que en las personas asignadas a $80 \mathrm{mg} / \mathrm{día}$ de atorvastatina se observó una menor incidencia de ECV, en los individuos con un elevado riesgo basal de diabetes asignados a 80 $\mathrm{mg} /$ día de atorvastatina se observó una mayor incidencia de esta condición clínica que en los asignados a bajas dosis, efecto no observado en las personas con bajo riesgo basal de diabetes.

Palabras clave: Atorvastatina, estatinas, incidencia, diabetes, evento cardiovascular. Fuente de financiamiento: No referida.

\section{Comentario}

Si bien la reducción de la incidencia de ECV con estatinas está ampliamente demostrada en prevención primaria y secundaria ${ }^{1,2}$; un reciente meta-análisis ${ }^{3}$ documentó que, comparadas con placebo, las estatinas aumentan un $9 \%$ el riesgo de desarrollar nuevos casos de diabetes. Por otro lado y en comparación con las dosis moderadas de estatinas, el tratamiento intensivo en altas dosis, lo incrementa un $12 \%$ adicional ${ }^{4}$.

Aunque los mecanismos no han sido aún aclarados, se han identificado algunos factores predisponentes para desarrollar diabetes, como los analizados por Waters y col. en el presente artículo ${ }^{5}$. Desconocemos si todas las estatinas son iguales. Por ejemplo, en el estudio JUPITER la rosuvastatina produjo significativamente más casos de diabetes en comparación al placebo; y en el WOSCOPS, la pravastatina produjo el efecto contrario. La pitavastatina, una nueva estatina, parecería tener un efecto neutral. De todos modos, deberíamos poner esta información en un contexto más amplio y contemplar el beneficio clínico neto, balanceando el riesgo de desarrollar nuevos casos de diabetes con la impactante reducción del riesgo de ECV. EI meta-análisis anteriormente citado ${ }^{3}$ documentó que si tratamos durante diez años 255 pacientes con estatinas, evitaremos nueve ECV y generaremos un nuevo caso de diabetes ${ }^{3}$; mientras que el tratamiento intensivo con estatinas (en comparación con el de dosis moderadas), reduce ${ }^{6,5} \mathrm{ECV}$ a expensas de tres nuevos casos de diabetes cada 1000/pacientes/año4.

\section{Conclusiones del comentador}

El presente estudio agrega la información de que el mayor riesgo de desarrollar diabetes asociado al tratamiento con dosis altas de atorvastatina sólo se observa en quienes tenían basalmente al menos dos factores de riesgo para desarrollarla, subpoblación que vemos con mucha frecuencia en nuestro consultorio. Consideramos que de todos modos deberíamos ofrecerles tratamiento con estatinas, explicándoles que existe alta probabilidad de que se su beneficio neto sea positivo, dada la categórica reducción de ECV mayores asociada a este tratamiento.

Walter Masson [ Servicio de Cardiología del Hospital Italiano de Buenos Aires. walter.masson @ hospitalitaliano.org.ar ]

*Ver glosario

Masson W. Efecto del tratamiento con atorvastina sobre la incidencia de eventos cardiovasculares y de diabetes. Evid Act Pract Ambul Oct-Dic 2013; 16(4):135. Comentado de: Waters D y col. Cardiovascular event reduction versus new-onset diabetes during atorvastatin therapy. J Am Coll Cardiol 2013;61:148-52.

\section{Referencias}

1.Taylor F y col. Statins for the primary prevention of cardiovascular disease. Cochrane Database Syst Rev. 2013 Jan 31;1.

2-Baigent C y col. Cholesterol Treatment Trialists' (CTT) Collaborators. Efficacy and safety of cholesterol-lowering treatment: prospective meta-analysis of data from 90,056 participants in 14 randomised trials of statins. Lancet. 2005;366:1267-78.

3-Sattar N y col. Statins and risk of incident diabetes: a collaborative meta-analysis of randomised statin trials. Lancet. 2010;375:735-42.

4-Preiss D y col. Risk of incident diabetes with intensive-dose compared with moderate-dose statin therapy: a meta-analysis. JAMA 2011;305:2556-2564.

5-Waters D y col. Cardiovascular event reduction versus new-onset diabetes during atorvastatin therapy. J Am Coll Cardiol 2013;61:148-52. 